\title{
DiOTTOS
}

Revista de Comunicación Digital

\section{¿De qué color es tu corazón? El uso de emojis en los procesos de activismo social}

\section{What color is your heart? The use of emojis in social activism processes}

Lucía Cantamutto*

Universidad Nacional de Río Negro

Centro Interdisciplinario de Estudios sobre

Derechos, Inclusión y Sociedad (CONICET)

\section{Resumen}

Este trabajo analiza el empleo de ciertos emojis como insignias de afiliación grupal en procesos de activismo digital. Centramos nuestro interés en la observación de diferentes perfiles de Twitter que presentan una actividad comunicativa relacionada con el debate en torno a la ampliación de los supuestos legales de la ley del aborto, que se produjo en las instituciones argentinas en junio de 2018. A través del rastreo de ciertos hashtags, observamos un proceso de remediación digital: el pañuelo verde como insignia de las movilizaciones callejeras de las activistas en favor de la despenalización del aborto se convierte en el emoji del corazón verde. De forma paralela, los detractores de esta reforma se agrupan en las redes sociales en torno al emoji del corazón azul. Los resultados de este trabajo confirman que algunos emojis funcionan como señas de identidad en la interacción digital y permiten manifestar la afiliación ideológica del usuario y del contenido generado por este. Esta función novedosa de los emojis es una estrategia propia de la dinámica actual del estilo digital.

\section{Palabras clave}

emojis, activismo social, identidad, Twitter, discurso digital

\section{Abstract}

This article analyses the use of certain emojis as an insignia of group affiliation in digital activism processes. We studied various Twitter profiles in which communicative activity revolves around the debate about the extension of the legal assumptions regarding the abortion law in Argentina in June 2018. We tracked certain hashtags and observed how they link to a process of digital remediation: green handkerchiefs becoming both the insignia of activists' street mobilizations in favor of abortion decriminalization and also the emoji of green hearts in social networks. At the same time, detractors of this reform get grouped in them using the blue heart emoji. The results of this study confirm that some emojis serve as signs of identity in digital interaction and also that allow users to express ideological/political affiliation as well as their own content. This new function of emojis is an inherent strategy of digital style.

\section{Keywords}

emojis, social activism, identity, Twitter, digital discourse

Recibido: 14/12/2019

Aceptado: 17/03/2020

*El orden de las autoras es alfabético. Ambas han colaborado por igual en la investigación.

Cómo citar este artículo:

Cantamutto, L. y Vela Delfa, C. (2020). ¿De qué color es tu corazón? El uso de emojis en los procesos de activismo social. Dígitos. Revista de Comunicación Digital, 6: 119-136. DOI: 10.7203/rd.v1i6.183 


\section{¿De qué color es tu corazón?}

El uso de emojis en los procesos de activismo social

\section{Introducción}

El activismo social ha encontrado en diferentes redes sociales de Internet nuevos espacios para la difusión y el desarrollo de sus posturas y propuestas. De hecho, muchas de las discusiones de la arena política se transfieren a Twitter y, aprovechando su sintaxis, se convierten en hashtags que sirven para ordenar los argumentos en uno y otro sentido. Internet favorece la organización de grandes coaliciones ideológicas que se ven dotadas de instrumentos muy potentes para conducir la acción colectiva. Esta puede concretarse en dos vertientes -offline y online- que, lejos de separarse, se retroalimentan mutuamente. Tal es el caso de estudio que nos ocupa en este trabajo.

El día 14 de junio de 2018 los habitantes de la República Argentina siguieron de cerca el debate en la Cámara de Diputados en torno al Proyecto de Ley de Interrupción Voluntaria del Embarazo. Tras más de 22 horas de intercambio de opiniones, se aprobó el proyecto que preveía la despenalización del aborto en ese país. Sin embargo, en su paso por la Cámara de Senadores, este no obtuvo la mayoría de los votos para su ratificación.

En este contexto, los meses previos a los debates y los posteriores a su rechazo estuvieron marcados por la movilización de diferentes grupos sociales que fueron generando insignias identificativas. Una de ellas fue el pañuelo verde. Retomando el símbolo de la histórica lucha que protagonizaron en Argentina las Abuelas de Plaza de Mayo (Sutton y Borland, 2017), el pañuelo se convirtió en una marca de cohesión entre quienes reclamaban la aprobación de este proyecto. La campaña por el Aborto legal, seguro y gratuito fue en el 2018 la promotora de la utilización de esta insignia; sin embargo, su creación data del Encuentro nacional de mujeres del año 2003. La utilización del color verde en las pañoletas provocó la denominación "marea verde", por metonimia, para el conjunto de las personas que se visibilizan en cada marcha en apoyo a la legalización del aborto en Argentina.

La discusión en torno al Proyecto de Ley de Interrupción Voluntaria del Embarazo en Argentina produjo una polarización entre quienes estaban a favor y en contra. Como ya hemos mencionado, las personas que apoyaron la legalización del aborto se identificaron con el color verde. Por su parte, quienes rechazaban el proyecto se identificaron con el color azul.

La batalla entre los defensores y detractores de la despenalización del aborto en Argentina fue más allá de las calles y tiñó también las cuentas de las redes sociales de las activistas. Tal y como sostienen Millaleo y Velasco (2013: 9), Internet ha sido usada para hacer aparecer nuevas formas y actividades de protesta online y para crear versiones virtuales de acciones de protesta que surgieron offline. Así, la dinámica de los colores se traslada a la actividad en redes sociales, a través de fotos, de videos y otros recursos multimodales. Con ellos, no solo se logra marcar la identidad colectiva, sino que también se consigue canalizar gran parte del componente emocional de estos intercambios comunicativos. 
Entre los recursos multimodales utilizados, cabe destacar el empleo de los emojis. Los emojis son un conjunto de íconos e imágenes prediseñadas, muy empleadas en la comunicación digital, para canalizar gran parte de la expresividad en la interacción digital escrita. De hecho, funcionan como claves de contextualización de los enunciados y estrategias de encuadre (Gallardo Paúls, 2014). Así el enfrentamiento online entre defensores y detractores del Proyecto de Ley de Interrupción Voluntaria del Embarazo se materializó en el uso de dos signos contrapuestos: el emoji del corazón verde y el emoji del corazón azul. Estos aparecen de forma recurrente tanto en los mensajes producidos en diferentes plataformas, como en la descripción de los perfiles de ciertas cuentas.

El objetivo de este trabajo es estudiar el empleo de estos emojis en la red social Twitter, como elementos clave para la gestión de la identidad digital (Pohl, Domin y Rohs, 2017). Para ello, llevamos a cabo una observación de diferentes perfiles de activistas de ambos grupos, con el fin de identificar qué emojis utilizan en las descripciones de sus cuentas y en los tuits publicados en los dieciocho meses siguientes al debate. Para la selección de los perfiles, hicimos un rastreo manual de los hashtags \#AbortoLegal o \#AbortoLegalYa y \#SalvemosLasDosVidas o \#SalvemosLas2Vidas.

Consideramos que el punto de vista abordado en este trabajo puede resultar interesante puesto que identifica un uso novedoso de los emojis, que podría añadirse a las clasificaciones clásicas de estos elementos (Sampietro, 2106b; 2019a; 2019b; Yus, 2014). Además, nos permite aportar pistas que sirven para explicar los procesos de semantización de los elementos multimodales y, lo que es más importante, datos sobre cómo estos se transforman en estrategias discursivas con valor expresivo, imprescindibles para la construcción y la gestión de la identidad digital.

Organizamos este trabajo en las siguientes secciones. Tras una introducción en la que se abordan los objetivos y la justificación de la relevancia del estudio, abrimos una segunda sección dedicada a presentar los principales conceptos teóricos manejados. La tercera parte se dedica a una presentación y discusión de los datos y se cierra con una cuarta sección, en la que se exponen las conclusiones.

\section{Los emojis como recurso expresivo en la red social Twitter}

Cada situación de comunicación ofrece a los interlocutores recursos particulares para canalizar la expresividad, característica inherente de la comunicación (Leech, 1986). En las redes sociales, estos recursos evolucionan muy rápidamente. Las interacciones digitales han ido incorporando progresivamente un conjunto de recursos multimodales (Herring, 2015; Lyons, 2014, Danesi, 2017), emojis, GIFs, memes, stickers, entre otros, que se emplean para favorecer la negociación y ajustar las interpretaciones desde el punto de vista expresivo.

En el caso particular de Twitter, los mecanismos de expresividad se adaptan a su particular arquitectura y sintaxis. Twitter es una red social de microblogging en la que se generan contenidos, en forma de publicaciones breves o tuits, y relaciones, a través del botón seguir.

El espacio identitario que ofrece Twitter está conformado por un perfil personal de carácter público, en el que se incluye un nombre de usuario y una breve descripción, una foto de perfil y una foto de portada. Además, este perfil contiene el número de seguidores e hipervínculos hacía las cuentas que se sigue. Cada perfil está asociado al espacio de interacción en que se integra la actividad enunciativa: los mensajes de su autoría, aquellos a los que ha reaccionado, aquellos que ha citado (mediante la acción 
de retuitear) y aquellos en los que es nombrado. Todos ellos de carácter público y ordenados de forma cronológica, en lo que se conoce como el timeline.

La sintaxis de Twitter habilita tres alternativas en sus enunciados. Por un lado, el uso de la @ (arroba), que sirve como hipervínculo apelativo para etiquetar a otros personas; el \# (hashtag), que se utiliza para identificar en qué conversación y/o tema se está participando, y el RT (retuit), que permite generar interacción entre los usuarios. Además de la publicación de tuits aislados, en la actualidad, se pueden construir hilos, o conjuntos de tuit interdependientes y conectados de manera jerárquica.

La utilización de elementos multimodales con valor expresivo es una tendencia que se acentúa progresivamente en todas las redes sociales y, también, en Twitter. Las fotos o los GIF animados acompañan, en no pocas ocasiones, a los tuit que se publican en esta red social. Por su parte, como tendremos ocasión de ilustrar, en las descripciones de los perfiles, se incluyen, mayoritariamente, emojis.

Los enunciados se adaptan a los recursos proporcionados por cada aplicación, al tiempo que se desarrollan estrategias novedosas, no siempre contempladas por el diseño original de las interfaces, y que, muy a menudo, se generan por contagio de los recursos empleados en otros entornos digitales. En Twitter, los emojis cumplen, como en otras plataformas, muchas funciones ligadas a la comunicación de la expresividad. Estos elementos se presentan como una alternativa más entre los diferentes recursos multimodales para dotar tanto de expresividad como de claridad a un enunciado. Esta dinámica, descrita en Vela Delfa (2007) y Cantamutto (2018), busca el equilibro entre los recursos expresivos, que doten de claridad al mensaje, y las estrategias de economía. Los emojis son, en esta lógica, candidatos a cubrir diferentes necesidades comunicativas.

El estado actual del discurso digital no puede ser pensado en los mismos términos que el previo a la convergencia digital. Las diferentes plataformas y aplicaciones se retroalimentan entre sí, de manera que esta integración también se concreta en la aproximación de diversas prácticas comunicativas y estrategias expresivas (Cougnon y Fairon, 2012). Así, por ejemplo, el botón me gusta en Facebook encuentra su equivalente en las interacciones por WhatsApp en el emoji thampietro, 2016a). De forma parecida, en las diferentes plataformas, se han desarrollado estrategias para mostrar acuerdo que, progresivamente, se han ido aproximando, no solo funcionalmente sino formalmente. En el caso de Twitter, el botón fav, cuya función es similar al me gusta, ha evolucionado formalmente desde una estrella a un corazón rojo, ícono que se parece al empleado en otras redes sociales, como Facebook.

Más allá de estas dinámicas de convergencia, en este trabajo nos interesa describir el uso de los emojis como canalizadores de estrategias de expresividad compleja, que responden a la necesidad de los usuarios de identificar sus perfiles con ciertas posturas discursivas. Se trata del empleo de los emojis como insignias identificativas en el activismo social digital.

En el caso concreto que nos ocupa, la incorporación del emoji del corazón verde o el emoji del corazón azul, respectivamente, se convierte en una marca de afiliación con una postura concreta en el debate sobre el Proyecto de Ley de Interrupción Voluntaria del Embarazo en Argentina. Esta tendencia se inserta dentro de una más general de resemantización de ciertos emojis, en tanto que emblemas de diferentes movimientos sociales. Estos signos pueden variar en función de diferentes factores, por ejemplo, 
el país: el corazón verde, en España, se puede encontrar actualmente en perfiles que quieren mostrar su identificación con el partido político Vox. Es por ello que, para rastrear de forma eficiente su valor como insignia, debe combinarse su empleo como el de otras señas, por ejemplo, con otros emojis o con algunos hashtag, que denotan la participación en ciertos temas. En cualquier caso, todas ellas funcionan como estrategias para la construcción de la identidad virtual en los perfiles de Twitter.

Para la comprensión de este fenómeno acudimos a la noción de identidad, reflejada en las propuestas de la microsociología. Goffman (1963) distingue la identidad personal de la identidad social. La identidad personal se construye a partir de la idea de unicidad: cada persona es una y distinta de todas las demás, una combinación particular de los ítems de la historia vital. La identidad social, por su parte, se corresponde con el concepto del "yo" que cada individuo construye en relación a los grupos sociales a los que pertenece, con los que se identifica (Capuano, Lucilli y Szwarc, 2008). En tal sentido, la inclusión de emojis insignia en los perfiles de Twitter colabora en la elaboración de la identidad social.

Para explicar esta construcción identitaria, Goffman (1959: 83) emplea la analogía teatral según la cual "la relación social común está de por sí organizada como una escena, con intercambio de acciones teatralmente infladas, contrapuntos y réplicas finales". Para Goffman, nuestra imagen depende de los otros, de cómo estos nos ven, nos representan y se relacionan con nosotros. Por ello, la imagen se concibe como el resultado de una elaboración (face work) que se va perfilando en el transcurso de la interacción: puede mejorarse, dañarse, transformarse, etc. Así concebido, el individuo construye su self a partir de su interacción con el otro, en buena medida, a través de la interacción verbal. Por lo que, también, la actividad comunicativa puede compararse con una escena, en la que distintos actores interpretan un papel o rol.

En sus diferentes trabajos, Goffman $(1971 ; 1983)$ trata de caracterizar los mecanismos de expresividad del individuo y, para ello, diferencia dos tipos de información: la que se da y la que se emana. La primera tiene que ver con los símbolos verbales, controlados por el individuo, como el lenguaje o los gestos, y la segunda con los no verbales, sintomáticos, no controlados. Podemos adaptar esta distinción entre lo expresado intencionalmente y lo expresado de manera involuntaria para explicar ciertos procesos de construcción de la identidad en el discurso digital, particularmente, en las redes sociales.

Así, por ejemplo, en Vela Delfa (2014) fue aplicada al análisis de los canales de Chat de IRC (Internet Relay Chat). Distinguimos entre la información dada, la que se mostraba de forma explícita en el nombre o perfil de usuario, que se correspondía con la información que los usuarios proporcionaban de manera voluntaria, y la información emanada, que se generaba de forma no controlada y que se implicaba, por ejemplo, de su presencia en determinados canales de IRC, de las relaciones jerárquicas que establecen entre sí los interlocutores o de las acciones sintomáticas que se llevan a cabo en el transcurso de la interacción.

Steingress-Carballar (2019) emplea esta distinción entre lo dado y lo emanado para analizar el valor de los selfies como parte del cyberselfy el cybercuerpo que "se constituyen como una extensión online del self-cuerpo offline". Cuestiona la aparente pobreza de la información implícita o emanada en las interacciones online y afirma que "si bien es cierto que la expresión emanada ya no se da, principalmente, a partir del lenguaje corporal, sigue habiendo un intercambio de información implícita e inconsciente por otros medios" (Steingress-Carballar, 2019: 134). 
Como veremos en nuestro análisis, esta distinción puede aplicarse con éxito al estudio de la identidad en la red social Twitter, a partir de la oposición que se produce entre el supuesto control que los usuarios tienen de la información que incluyen en sus perfiles (fotos, descripciones, etc.), y la información que se emana inconscientemente, a través de escenarios o expresiones implícitas que el receptor percibe en la interacción.

La información emanada actúa como una especie de marco, es decir, de fondo, sobre el que se proyecta la información dada. Esta última es el resultado de un proceso continuo de co-construcción, que se realiza sobre la base de la información obtenida en la interacción. De tal suerte que la construcción de la identidad es un proceso dinámico que consiste, más que en la creación de un personaje, en su continua recreación, en el proceso de interacción. En este sentido, un locutor recrea cada una de sus identidades cada vez que participa en una interacción.

En redes sociales como Twitter, la oposición entre información dada e información emanada se concreta en la diferencia entre, por un lado, la información que se incluye en los perfiles de los usuarios, en los hashtag que utilizan y en las redes de cuentas seguidas y, por otro, la información aportada en el transcurso de las interacciones, en los distintos mensaje que publican. Así, la información ofrecida en los perfiles sirve para presentar al enunciador y permitir que el co-enunciador establezca hipótesis que irán confirmándose en el proceso de interacción. En el caso de Twitter, al tratarse de una red pública, además, toda la construcción discursiva se ve sometida a procesos de resignificación, a través de diversos procedimientos de cita, entre los que destacan los retuit o las reacciones directas a partir del botón me gusta. Todas ellas producen un cierto efecto eco en la enunciación.

\section{Diseño metodológico y presentación de los datos}

El objetivo de este trabajo es el análisis del uso de los emojis de corazón verde y corazón azul como insignias que identifican la participación de algunos perfiles de Twitter en movimientos de activismo social en la red, particularmente, en lo que concierne al Proyecto de Ley de Interrupción Voluntaria del Embarazo en Argentina. Nuestra hipótesis de partida sostiene que estos elementos, que configuran una parte fundamental de la información dada (en el sentido goffmaniano del término), resultan fundamentales en la configuración del encuadre discursivo e ideológico de los perfiles. Para ello, debemos verificar qué usos se hacen de estos emojis y si existe alguna correlación con ciertas posturas discursivas. La conjetura que sostenemos es que, si bien existen usos individuales de estos emojis, en un número relevante de ocasiones sirven para manifestar un proceso de identificación grupal.

Desde el punto de vista metodológico, tres son las principales dificultades con las que nos hemos topado en este trabajo: la primera tiene que ver con la selección de los datos, la segunda, con la pérdida de elementos paratextuales en los procesos de extracción masiva de datos, y la tercera, con una cuestión de tipo ético.

Para comenzar la investigación, necesitábamos documentar muestras del empleo de estos emojis en relación al debate sobre Proyecto de Ley de Interrupción Voluntaria del Embarazo y separarlos de otros valores. Existen muchas herramientas y APIs que permiten extraer datos de Twitter e, incluso, desde la misma red social es posible realizar pesquisas. La estrategia metodológica empleada fue seleccionada tras la puesta a prueba de alternativas subsidiaras que nos permitieron ajustar el modelo empleado. La primera técnica utilizada consistió en utilizar la herramienta \# Explorar 
de Twitter que se identifica, visualmente, con los hashtags. En la barra de búsqueda, se inscriben los términos de la indagación, que se pueden filtrar por ubicación. Además, se ofrecen subsecciones para explorar tendencias personalizadas (para ti), o por secciones (noticias, deportes, diversión y entretenimiento). Si lo que se quiere buscar es un emoji, como en nuestro caso, la aplicación móvil será más adecuada, ya que el teclado del teléfono ofrece la posibilidad de escribir directamente el emoji en el buscador; desde la interfaz del ordenador, sin embargo, tendríamos que proceder a escribir un tuit con el emoji y, luego, copiarlo para pegarlo en la caja de búsqueda de Twitter. Mediante esa técnica, realizamos dos búsquedas (una por cada color de corazón). Lo resultados que nos devuelve la red social son muchos y muy variados. Esta abundancia y disparidad constituye el primero de nuestros problemas metodológicos.

Tras la revisión de estos primeros resultados, observamos dos tendencias generales, en relación al empleo de los emojis: 1) las ocurrencias que se corresponden con un uso significativo consciente, generalmente, integradas en los perfiles y 2) las ocurrencias que muestran un uso más lúdico u ornamental, y que no pueden incluirse en una función identitaria.

Entre los usos significativos controlados, encontramos también gran variedad. Por ejemplo, el corazón verde está extendido entre los ecologistas o los votantes o simpatizantes del partido político español Vox. Además asumen un sinfín de significados que emergen a diario y que pueden asignarse a la combinación del corazón, elemento expresivo, con el color verde o azul. La aplicación ofrece, al momento de realizar la investigación, como búsquedas predefinidas las colocaciones de

La diversidad y viralización temporal de estos usos puede ilustrarse con dos casos concretos, que surgieron durante el periodo de desarrollo de este trabajo. El primero se corresponde con el empleo del emoji del corazón verde para celebrar el mantenimiento de un jugador emblemático del Real Betis Balompié (equipo español de futbol). Debe tenerse en cuenta que el verde es el color de este equipo. De hecho, en el perfil de Twitter del Real Betis Balompié, acompañando al nombre del club, se incorpora un emoji de corazón verde: “Real Betis BalompiéT @ @RealBetis". El segundo tiene que ver con la propuesta del empleo del emoji del corazón verde como elemento identificativo de la defensa de las escuelas de educación especial en España. Así se reivindicaba en algunas cuentas:

(1) Os invito otra vez a que os pongáis el que quiere decir NO AL CIERRE DE LOS COLEGIOS DE EDUCACIÓN ESPECIAL ${ }^{1}$

En el caso del emoji de corazón azul, también encontramos usos que van más allá del debate para la despenalización del aborto. Así, se observa su combinación con otros emojis para representar la bandera del equipo argentino de Boca (véase Ejemplo 12), cuyo perfil oficial. Sin embargo, @BocaJrsOficial, no emplea este elemento.

Los usos que emergen en una búsqueda por emojis no ofrecían evidencias de su función identificativa con el movimiento a favor o en contra del Proyecto de Ley de Interrupción Voluntaria del Embarazo. Por lo tanto, la siguiente estrategia fue realizar una búsqueda complementaria a partir de cuatro hashtags, a fin de verificar esta relación. Realizamos dos llamadas durante el mes de diciembre de 2019, en las que buscamos los hashtag \#AbortoLegaly\#AbortoLegalYay, luego, \#SalvemosLasDosVidas o \#SalvemosLas2Vidas. Los resultados se transcribieron en una planilla de Excel, seleccionando, en cada una, los

1. Los tuits que se recogen en este trabajo respetan la redacción original. 
primeros cincuenta perfiles que, aleatoriamente, ofrece la plataforma y los cincuenta tuits destacados que brindaba Twitter. Este procedimiento se hizo para sendos pares de hashtag.

Así, el conjunto de datos analizado se corresponde con una muestra de cien perfiles implicados de forma directa en el debate sobre el Proyecto de Ley de Interrupción Voluntaria del Embarazo y un corpus de cien tuits destacados. Si bien existen numerosas herramientas para la extracción masiva de datos, esta alternativa nos pone frente a nuestro segundo problema metodológico. Es posible recopilar de forma sistemática todos los mensajes publicados con las etiquetas seleccionadas (los hashtag, en este caso). Sin embargo, al proceder a la descarga automática de los tuits, se pierde información que resulta crucial para nuestro análisis. La recuperación de los emojis resultaba parcial, se perdía, por ejemplo, el color, por lo que no nos era posible diferenciar el uso de un emoji de otro. Por ello, optamos por la descarga manual. Esto nos obligó a trabajar con un corpus de tamaño reducido pero muy denso desde el punto de vista informativo.

Por último, la tercera de las dificultades con las que nos topamos en esta investigación es de carácter ético. Twitter permite la obtención de gran cantidad de datos que, a priori, pueden considerarse públicos. Así lo recuerda Moreno-Ortiz (2019: 61) "la política de privacidad de Twitter deja bien claro esta transparencia de sus datos ya que todos los contenidos son públicos y abiertos". Sin embargo, desde nuestro punto de vista, y teniendo en cuenta la dificultad para conseguir el consentimiento informado de todos los individuos que están detrás de los perfiles observados, hemos preferido implementar algunas estrategias de anonimización.

Todos los ejemplos citados tienen por objetivo ilustrar la utilización del emoji de corazón verde y del corazón azul como elementos insignia en los diferentes perfiles. Sin embargo, desde un posicionamiento ético, no presentamos los enunciados completos sino que referimos únicamente fragmentos que reflejan los contextos relevantes y las colocaciones más usadas. Además, para evitar el uso de capturas de pantallas que no permite la anonimización y que, además, por la trazabilidad de los datos, favorece la identificación de los usuarios, consideramos que una solución metodológica apropiada consiste la generación de ejemplos construidos, a partir de la mezcla de publicaciones reales (de-Matteis, 2016). Con esta técnica es posible ilustrar los usos y evitar la exposición de los autores de los tuits, que no fueron publicados con el fin de formar parte de un estudio de investigación como el que nos ocupa.

Luego de un análisis exploratorio de los datos extraídos, hemos optado por una técnica que nos permitiera realizar un análisis en profundidad de los perfiles y las cuentas seleccionadas aleatoriamente por el algoritmo de búsqueda de Twitter. Para verificar la relación entre los hashtag y los emojis, hemos empleado el diagrama de Sankey que favorece la visualización de esta vinculación.

\section{Análisis y discusión de los datos}

A medida que se expande el repertorio de emojis disponibles en aplicaciones y teclados y que su uso se extiende en diferentes contextos, se producen dos fenómenos complementarios. Por un lado, tanto los usuarios como los medios de comunicación buscan establecer consensos y acuerdos sobre su significado. Por otro lado, el empleo real que los usuarios hacen de estas unidades conquista cada día un abanico de funcionalidades más amplio, lo que dificulta la atribución de interpretaciones unívocas.

Desde una perspectiva pragmática, son las personas usuarias quienes definen 
el significado contextual de cada una de estas imágenes, cuyo valor está anclado tanto socioculturalmente como contextualmente. Estos valores se establecen por combinación con otros elementos, con los que entablan una relación de correlación. En algunas ocasiones, pueden adquirir un alto grado de estandarización hasta convertirse en insignias representativas y distintivas de un determinado movimiento discursivo. Este es el caso de los emojis con forma de corazón. Su evidente dimensión emocional, relativa a la iconicidad de su forma, los ha convertido en un recurso expresivo muy empleado en Twitter. En sus diferentes colores, rojo, verde, azul, violeta, amarillo, el emoji de corazón ha sido resemantizado por distintas corrientes ideológicas que fluyen en Twitter. De tal marera que estos corazones se han convertido en marcas de expresividad dada, según la definición de Goffman. De este modo, la variación del color de los emojis de corazones se erige en una función novedosa de los emojis: su uso como insignia, como emblema distintivo de una institución, asociación, o marca comercial, que se emplea como muestra de vinculación o simpatía.

La utilización del emoji de corazón verde y del emoji de corazón azul presenta diferencias en cuanto a su prevalencia y a su especialización. Como se desprende de los datos ofrecidos en una consulta a emojitraker ${ }^{2}$, aplicación que da cuenta a tiempo real del uso de los emojis en Twitter, ambos son emojis utilizados frecuentemente. No obstante, el emoji del corazón azul se emplea más frecuentemente, ocupa el puesto vigésimo sexto, frente al quincuagésimo sexto lugar ocupado por el emoji verde. Sin embargo, su empleo, en tanto que insignia es menor.

Así, en lo que concierne al emoji del corazón verde, su empleo como elemento de identificación ideológica está más extendido, en relación al número total de ocurrencias. De hecho, en cada una de nuestras búsquedas combinadas de emojis y hashtag, \#AbortoLegal y \#AbortoLegal, hemos comprobado que $\bullet$ arroja mayor cantidad de resultados. Estos nos permiten sistematizar su uso en relación con la lucha para la despenalización del aborto, a pesar de que conviva, como ya comentábamos más arriba, con otros usos estandarizados y no estandarizados. Por ejemplo, este tuit que expone una imagen de un pastel de manzana acompañado por este enunciado:

\section{(2) Buenas tardes, ahora toca merendar}

Por su parte, en las búsquedas relacionadas con el encontramos una importante dispersión; lo que nos lleva sospechar que su estandarización como elemento significativo en el debate sobre la despenalización del aborto se produce como reacción al emoji del corazón verde. Llama especialmente la atención el empleo de esos emojis en asociaciones divergentes: de hecho, el emoji de corazón verde y del corazón azul aparecen combinados en algunos perfiles, cuya información no está asociada a esta contienda social.

A continuación, presentamos el análisis de cien los perfiles seleccionados y de los tuits destacados. A lo largo de nuestra observación, hemos encontrado que, con frecuencia, los emojis del corazón verde y del corazón azul se incluyen tanto en las descripciones de los perfiles, como en los tuits. Podemos identificar tres estrategias básicas:

1) El empleo del emoji como parte del nombre de perfil y/o en la descripción de la cuenta. Cuando aparece acompañando al nombre del usuario no es raro que se coloque detrás de este o enmarcándolo, es decir, tanto al principio como al final. 
2) El empleo del emoji en el tuit, sin que este aparezca en el perfil de usuario. En tales casos, nos encontramos con perfiles menos identificados con activismo social que quieren, sin embargo, marcar ideológicamente algunas de sus contribuciones.

3) El empleo del emoji tanto en el nombre de usuario como en el tuit. En algunas ocasiones hemos encontramos este empleo redundante del emoji del corazón verde, que podríamos considerar sirve para enfatizar la adhesión ideológica de mensaje.

\subsection{Análisis de los perfiles}

Como decíamos más arriba, para la realización de este trabajo hemos identificado cien perfiles (cincuenta para cada hashtag) que aparecen tras las búsquedas realizadas mediante los hashtag mencionados. La decisión de combinar las pesquisas a través del hashtag y del emoji está basada en la necesidad de determinar si existe correlación entre el uso de este elemento multimodal con los hashtags correspondientes, es decir, verificar la identificación del emoji con otro elemento de activismo digital.

En relación a esta distribución hemos encontrado algunas diferencias en función de los colores. Con una diferencia mínima, el corazón verde se emplea de forma más generalizada en los perfiles con compromiso activista, mientras que el corazón azul se identifica con perfiles más generales, dado que su empleo se corresponde con otras funciones no consensuadas. Es decir, la identificación activista de los perfiles en defensa de la despenalización del aborto es mayor. Así, hemos observado que en muchas cuentas institucionales, es decir, relativas a agrupaciones y no a personas individuales, la mayoría de los tuis carecen de emojis. Aunque estos aparecen de forma recurrente en la descripción de sus perfiles. Esto sucede a menudo con el emoji del corazón verde, empleado por cuentas colectivas de los movimientos feministas.

Como consecuencia de esta distribución, se reducen los usos de emojis ligados a la expresividad, para reforzar su empleo como insignia ideológica. De hecho, en los tuits producidos por estos perfiles, se emplea un número muy reducido de emojis. Encontramos casos de emojis-insignia (corazón violeta y corazón verde) y un repertorio de emojis gestuales, que funcionan metonímicamente para indicar lucha o fuerza $(b-5)$. Además, en las cuentas institucionales se encuentran con frecuencia emojis con valor indicial ( $\nleftarrow \downarrow \downarrow$ ). La mano con un dedo hacia abajo podría considerarse actualmente una suerte de elemento de la arquitectura de Twitter, cuya función es indicar la apertura de un hilo, o conjunto de tuits relacionados.

En el análisis de los cien perfiles seleccionados hemos comprobado una mayor presencia de emojis de corazón verde: el $56 \%$ de las cuentas destacadas que emplean los hashtags \#AbortoLegal y \#AbortoLegalYa también usan el emoji insignia. En el caso de los otros hashtags el porcentaje es un poco menor: el $50 \%$ de las cuentas lo hace en coocurrencia con el emoji insignia azul. De hecho, en las cuentas que usan el corazón verde, como parte de la información dada en el perfil, observamos que con frecuencia este se combina con la referencia directa a los hashtags. Los siguientes ejemplos, en los que se han omitido los nombres de los usuarios para evitar su identificación, encontramos distintas colocaciones que resultan muy representativas de las estrategias de encuadre empleadas por distintos usuarios.

(3) Nombre Apellido $『$ \#AbortoLegalYa $@$ @NombreApellido

(4) Nombre \#AbortoLegalYa @ @nombreapellido 
(5) \#AbortoLegal2020 Nombre @NombreApellido

El ejemplo (6) ilustra una combinación frecuente de dos emojis insignias: el corazón verde y el corazón violeta. Este último como insignia de la lucha feminista. El empleo de estos dos corazones juntos es muy frecuente en las descripciones de cuenta, como se ve a continuación, en el ejemplo (7).

(6) \#SeguimosEnCampaña @cuentadeasociación

(7) ¿Para que callar si nacimos gritando? Que sea ley Uso esta cuenta para discutir con providas nomá

Como las insignias que se colocan en la ropa, cada una de ellas identifica al usuario $y$, lo que es más importante, sirve para enmarcar discursivamente una determinada postura ideológica de los tuits que se publican en esa cuenta.

En algunas ocasiones, las insignias pueden sufrir ciertas variaciones, como en ejemplo (8) y (9).

(8) WESTAS EN ESTA VIDA PARA SER FELIZ Y VIVIR A TU MAYOR VIBRACIÓN, EN EL AMOR DIVINO.W

(9) Sobrenombre \#Salvemoslas2Vidas Vekle @InicialNombreApellido

El Señor sabe quién soy. Esto me basta... -Sn.Juan XXIII-

Sin embargo, si vinculamos el emoji con el contenido del enunciado, podemos suponer que las mariposas azules se han elegido por el valor simbólico de su color. Estos emojis pueden sustituir a la insignia, en tanto que alternativa (ejemplo 8), o bien, como sucede con la presencia del emoji de la ola azul $\mathbb{E}$ c (ejemplo 9), desempeñar la función de elementos de refuerzo del emoji insignia. Así mismo, en los perfiles correspondientes a ambas posturas, se emplean emojis de banderas (de Argentina, en este caso) como reemplazo del ítem léxico (Sampietro, 2019b), para reforzar el valor de los emojis de corazón.

La combinación de los emoji insignia de corazón verde y azul con otros emojis se asocia con la vinculación entre diferentes colectivos de activistas, generándose sinergias entre sus reivindicaciones. Así, como ya hemos ilustrado más arriba con los ejemplos (6) y (7), su coaparición con el corazón violeta vincula la lucha por el aborto con el movimiento feminista. En el ejemplo (10) podemos ver como el corazón verde, se asocia, también, con el colectivo LGBT. Por su parte, el corazón azul se relaciona con colectivos religiosos (ejemplo 11)

(10) feminist \#AbortoLegal @sobrenombreApellido_Feminista desde que entiendo \#LGBT la Yuta.

(11) Sobrenombre -salvemos las 2 vidas @SobrenombreApellido1 Católica AAmo la Música.Jy Amo hacer lo q se me da la gana ...

También llama la atención el empleo de forma combinada de ambas corazones, como en el ejemplo (12).

(12) \#AbortoLegalYa @xxx_Nombre

de Boquita y Peronista Mamá de Delfina. Lanús Militante Feminista de @la_campora Políticamente Incorrecta. @CFKArgentina Conducción 
Ahora bien, en estos casos solo uno de los emojis se emplea como insignia del debate sobre el aborto; el otro asumen otro valor identificativo. En este ejemplo concreto, el corazón azul se emplea como referencia a uno de los colores de la bandera de Boca Juniors, probablemente.

Para concluir la sección dedicada al análisis de los perfiles, realizamos un diagrama de Sankey (Ilustración 1). En el contabilizamos la totalidad de emojis utilizados por las cien cuentas analizadas y los organizamos en relación a entornos simbólicos recurrentes. Así, para facilitar la visualización de los datos, se contabilizan de forma conjunta las diferentes categorías.

Por ejemplo, en torno al concepto de diversidad, incluimos algunos emojis como la bandera arcoiris $=$ el arcoíris y otros como este que reproducimos a continuación:

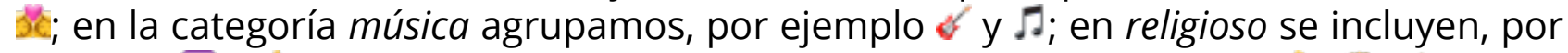
ejemplo, †o $\mathrm{d}$. En la categoría fuerza hemos incorporado tres gestos: $\mathbb{b}, \vec{b}$.

Por último, en la categoría random se han agrupado aquellos emojis que funcionan de manera lúdica, ilustrando, por ejemplo, diferentes aspectos de la vida cotidiana de los usuarios.

Para realizar estas agrupaciones, hemos tenido en cuenta no solo la imagen del emoji, sino el uso que se ellos se hace en contexto. De hecho, algunos de estos emojis podrían tener interpretaciones diferentes, si no se observara el contexto de utilización. Así,

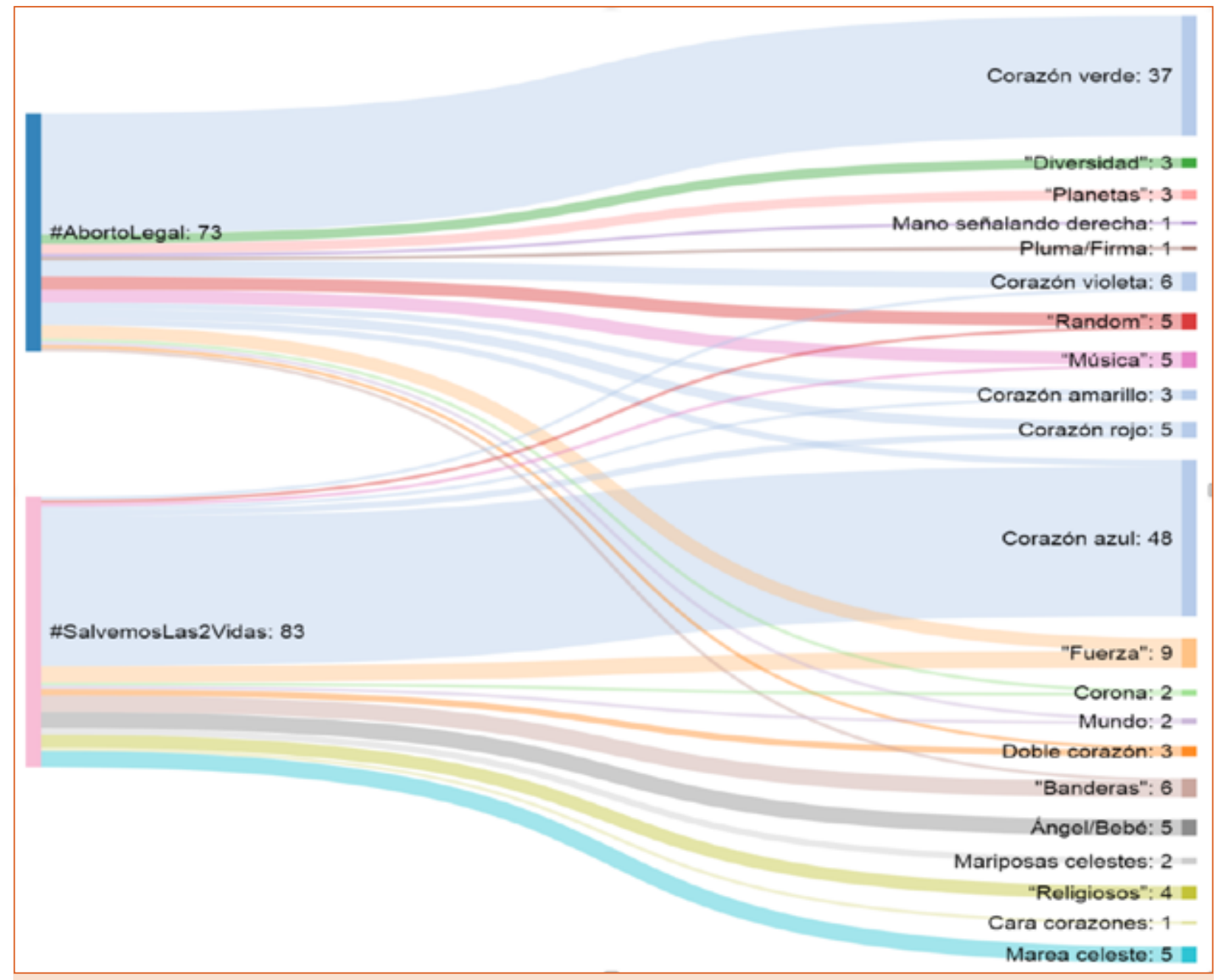

Ilustración 1. Cantidad de emojis presentes en los cien perfiles analizados. 
sucede, por ejemplo, con los emojis bै, $\$$ y. En nuestro análisis, por el contrario, su función es bastante homogénea y, en su mayoría, sirven para intensificar el valor de los emojis-insignia de corazón.

Si examinamos los datos cuantitativos que se extraen de nuestro análisis, podemos observar que el segundo grupo manifiesta un empleo más profuso de emojis. Mientras los relativos al hashtag \#AbortoLegal y \#AbortoLegalYa contienen 73 emojis, los perfiles que incluyen en el hashtag \#Salvemoslas2vidas o \#SalvemosLasDosVidas emplean 83. También son más abundantes los corazones azules (46), en este segundo, grupo que los verdes (37) en el primero.

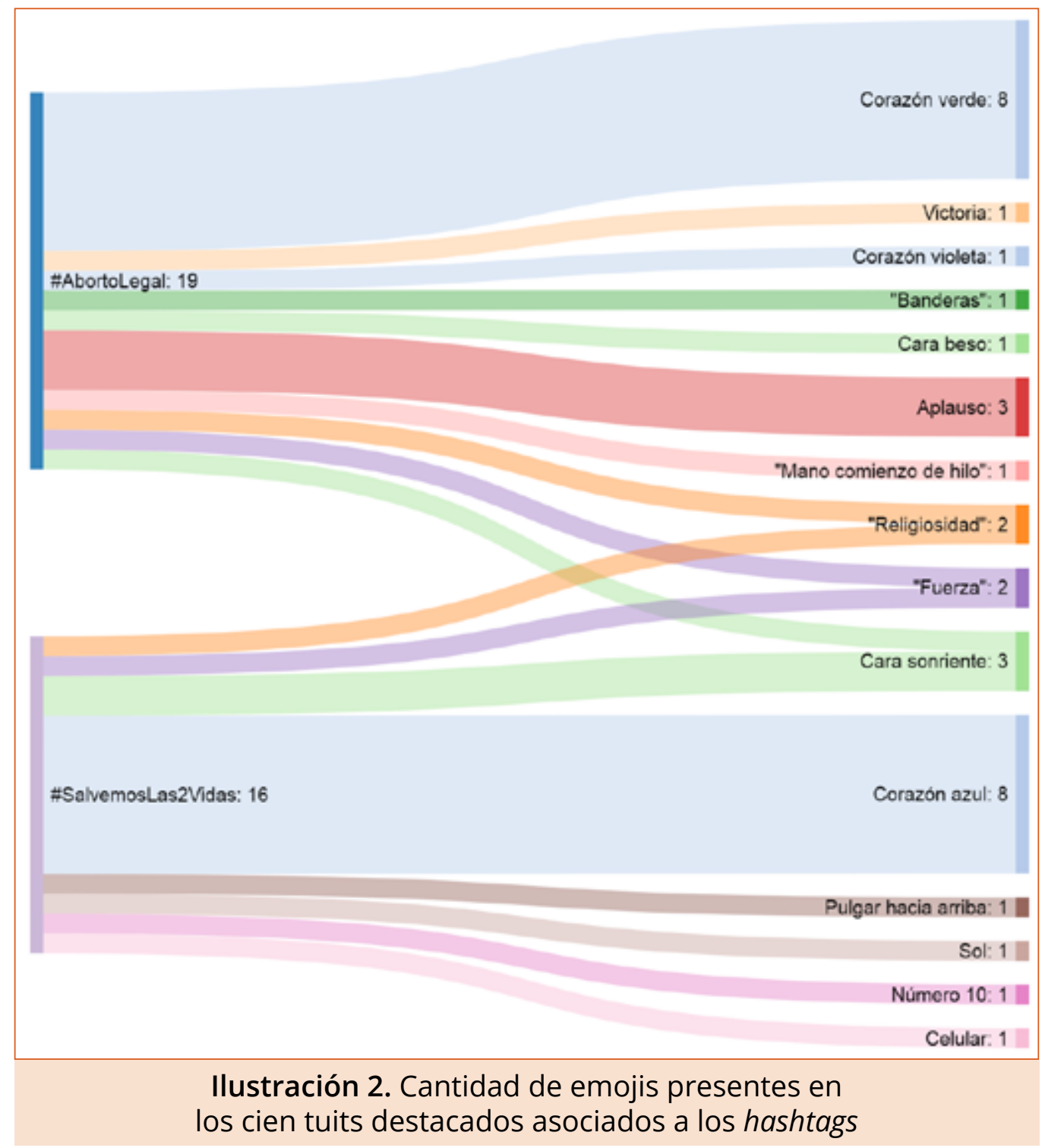

\subsection{Análisis de tuits destacados}

Una vez analizada la presencia de emojis insignia en los perfiles, pasamos a examinar su aparición en las publicaciones. Observamos que el diagrama de Sankey de los emojis utilizados en los cien tuits destacados presenta divergencias frente al análisis de los perfiles de los usuarios.

En primer lugar, se evidencia una menor presencia de emojis en los tuits recuperados que en los perfiles. Si bien en términos cuantitativos, hay mayor cantidad de palabras en la selección de tuits, hay un decrecimiento de la presencia de elementos multimodales. A 
pesar de ello, la función de emoji insignia sigue siendo una de las prioritarias. De hecho, casi la mitad de los emojis usados en relación a los hashtag analizados se corresponden con el corazón verde y corazón azul, respectivamente. Otras funciones que cubren los emojis inventariados tienen que ver con su dimensión expresiva, paralingüística y gestual; de hecho, aparecen las caras sonrientes y de besos. En el ejemplo (13) el empleo del emoji de aplauso, repetido en tres ocasiones, podría servir para intensificar, y se integraría en el subconjunto de emojis que cubren el valor semántico de fuerza o ánimo.

(13) \#AbortoLegal "La objeción de conciencia no puede ser una coartada. Lo que nosotros no queremos que esto se convierta en un combate", @usuario. Ya está en el Boletín oficial.

También llama la atención que entre los tuits recuperados se incluyan enunciados que contrargumentan la consigna representada por el hashtag que mencionen. Este es el caso ilustrado por el ejemplo (14). No obstante, lo llamativo es que, en estos casos, no hemos encontrado ningún emoji, lo que vendría a reforzar la hipótesis de que estos sirven como insignias y claves de contextualización discursiva. En tal sentido, resultaría poco apropiado emplearlos cuando la corriente ideológica es diferente.

(14) Esta cuenta se opone a la masificación efectiva del \#AbortoLegal libre y gratuito y a las \#retenciones a las exportaciones del campo por entender que ambas acciones conspiran contra una sana política económica. Es más, anticipamos graves problemas por ambos lados.

\section{A modo de conclusión: hacia una caracterización de los emoji insignia}

Tal como mencionamos en el apartado de la metodología, en este trabajo hemos empleado diversas técnicas de recogida de datos para analizar el empleo del emoji del corazón azul y el emoji del corazón verde, en relación al debate sobre el Proyecto de Ley de Interrupción Voluntaria del Embarazo en Argentina. Mediante este estudio exploratorio, buscamos verificar la hipótesis de que ambos emojis asumen la función de insignia. Como comprobamos en nuestro análisis, las cuentas que emplean los emojis en sus descripciones resultan más comprometidas ideológicamente y con un mayor compromiso activista.

Esta función de insignia, semejante a la que adoptan ciertos objetos físicos en las movilizaciones ciudadanas de las calles, refleja una doble dimensión: 1) sirve para manifestar la adhesión del enunciador con una determinada postura y, 2) funciona como clave de contextualización discursiva sobre la que pivota la orientación interpretativa de sus enunciados.

Esta doble funcionalidad puede justificar el hecho de que ambos emojis se empleen de forma más abundante en cuentas institucionales o colectivas que personales. $Y$, al mismo tiempo, sirva para explicar que, en las cuentas personales, estos emojis insignia se incorporan preferentemente en los tuits.

Aunque, el empleo de emojis en los enunciados se corresponde con un rasgo de estilo personal, es cierto que, en las cuentas que manifiestan una mayor adhesión a una determinada postura, los emojis se sitúan preferentemente en los perfiles. Es decir, forman parte de la información dada por los usuarios, según la distinción propuesta por Goffman. De esta forma, los emojis no actúan únicamente en tanto que claves de contextualización de uno (o varios) enunciados particulares, sino de la postura ideológica completa defendida por el usuario. En tal sentido, estos emojis funcionan 
como estrategias de encuadre en el sentido en que las definen Enguix Oliver y Gallardo Páuls (2014: 4) "en tanto que operaciones estratégicas de elaboración textual por las que se eligen los elementos discursivos que orientan cognitivamente la interpretación de un mensaje".

Así, por ejemplo, al analizar los perfiles de Twitter de las principales militantes de la Campaña argentina por el Aborto Legal, Seguro y Gratuito, hemos encontrado ciertas colocaciones, que combinan de forma recurrente hashtag y emojis.

El hashtag \#AbortoLegalYa, que ha sido uno de los más utilizados por las organizaciones y los actores que buscan legalizar el aborto, se combina con otros hashtags que ayudan a identificar el colectivo: \#NiUnaMenos.

Por su parte, la combinación de emojis también resulta recurrente. En ocasiones los emojis insignia se combinan entre sí, por ejemplo, el corazón verde y el corazón violeta aparecen de forma a reiterada en combinación. Resulta también repetida la combinación de los emojis insignia con otros emojis que orbitan en el campo semántico de la fuerza, por ejemplo $b$ y 3 . Estos emojis funcionan tanto como estrategias de intensificación de contenido ideológico marcado por la insignia, como indexadores ideológicos propiamente dicho.

La combinación de emojis insignia y hashtag también es usual. Algunas colocaciones frecuentes, en las cuentas en defensa del aborto, son, por ejemplo: \#SiAIAborto ,

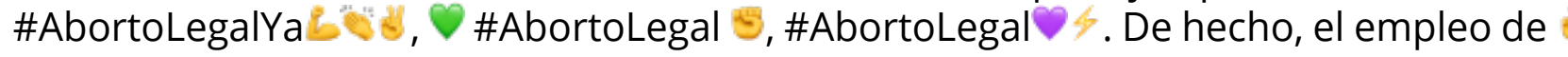
también podría considerarse con una función insignia ya que, asociado a determinados hashtag y contextos, suele ser un marca de afiliación con la figura política de Cristina Kirchner.

Como puede observarse, en la mayoría de estas combinaciones el corazón aparece antes y/o después del hashtag. Esta estrategia de coocurrencia de hashtags con emojis ha sido retomada por la propia sintaxis de Twitter, que la ha incorporado como un elemento particular de su repertorio expresivo. Este sería el caso de los hashflags, surgidos en el entorno de las competiciones deportivas, y promocionados por Twitter como una forma divertida de añadir color a los tuits. Se trata de hashtags especiales que tienen una pequeña imagen a su lado en los tuits que los incluyen.

Este trabajo exploratorio presenta diferentes vías de continuación. Por un lado, nos interesa, en un futuro, seguir explorando el empleo de emojis-insignia como clave de contextualización de las interacciones digitales. Esta función que no es exclusiva de la red social Twitter, dado que la hemos observado también en perfiles de WhatsApp y en las descripciones de Facebook. En todo caso, se trata del aprovechamiento de recursos digitales en determinados contextos enunciativos particulares que pueden ser habilitados por la arquitectura de las plataformas. El uso de emojis-insignia para indexar ideológicamente un perfil es un mecanismo económico que evita la indexación de cada uno de los enunciados producidos. Esto se ve, con claridad, en la merma de emojis presentes en los cien tuits destacados en relación con los perfiles.

Además, creemos que la función del emoji insignia es un elemento clave para comprender las estrategias pragmáticas que los usuarios emplean en sus interacciones digitales. La identificación de la cuenta mediante un emoji determinado funciona como clave de contextualización tanto del perfil como de todo el contenido producido por este. De hecho, la mecánica empleada para recoger los datos analizados confirma que en Twitter el empleo de emojis (al menos en los tuits) no es tan profuso como en otras 
redes sociales en las cuales la expresividad y el mantenimiento del contacto son más necesarios (como en WhatsApp). Este aspecto debería ser profundizado a la hora de abordar el estudio del discurso digital: si bien existen usos comunes a las diferentes aplicaciones, hay funciones que se asocian a determinada red social y comunidad de usuarios.

\section{Referencias}

Cantamutto, L. (2018): Estrategias pragmáticas de la comunicación por SMS en español bonaerense. Tesis Doctoral, Bahía Blanca: Universidad Nacional del Sur.

Capuano, A., Lucilli, P. y Szwarc, L. (2008): “Apuntes para la reflexión sobre el concepto de identidad", en Oficios Terrestres, $\mathrm{n}^{\circ} 21$, pp. 124-133.

Cougnon, L.-A. y Fairon, C. (2012): "Introduction". Lingvisticae Investigatione, vol. 2, n 35, pp. 155-162.

Crystal, D. (2001): Language and the Internet. Cambridge: Cambridge University Press.

Danesi, M. (2017): The semiotics of Emoji. London: Bloomsbury.

De-Matteis, L. (2016): “Ejes para una discusión del uso ético de datos interaccionales escritos y orales obtenidos en línea". En: Cantamutto, L., del Río, G. y Striker, G. (comp.) Actas de las 1eras Jornadas Nacionales de Humanidades Digitales: culturas, tecnologías, saberes. Buenos Aires: FyL-UBA.

Gallardo-Paúls, B. (2014): Usos políticos del lenguaje: Un discurso paradójico. Barcelona: Anthropos.

Gallardo-Paúls, B. \& Oliver, S. E. (2014): “Estrategias de open acc discursivo en periodismo político: análisis de un corpus de titulares", en Círculo de lingüística aplicada a la comunicación, $n^{\circ}$ 58, 90-109.

Goffman, E. (1959): The presentation of self in everyday life. Nueva York: Doubleday Anchor.

Goffman, E. (1963 [1989]): Estigma: la identidad deteriorada. Buenos Aires: Amorrortu

Goffman, E. (1971): Relations in public: microstudies of the public order. Nueva York : Basic Books.

Goffman, E. (1983): “The interaction order", en American Sociological Review, n 48, pp. 110-117.

Herring, S. C. (2015): "New frontiers in interactive multimodal communication". En Georgopoulou, A. y T. Spilloti (Eds.): The Routledge handbook of language and digital communication. London: Routledge, pp. 398-402.

Leech, G. (1986): Principles of Pragmatics. Singapore: Longman.

Millaleo, S. y Velasco, P. (2013): Activismo digital en Chile. Repertorios de contención e iniciativas ciudadanas. Santiago de Chile: Fundación Democracia y Desarrollo.

Pohl, H., Domin, C. y Rohs, M. (2017): "Beyond just text: semantic emoji similarity modeling to support expressive communication ", en ACM Transactions on Computer-Human Interaction (TOCHI), vol. 24, $\mathrm{n}^{\circ} 1,6$.

Sampietro, A. (2016a): “Emoticonos y multimodalidad. El uso del pulgar hacia arriba en WhatsApp", en Aposta. Revista de Ciencias Sociales, n 69, pp. 271-295.

Sampietro, A. (2016b): Emoticonos y emojis. Análisis de su historia, difusión y uso en la comunicación digital actual. Tesis Doctoral. Universidad de Valencia. 
Sampietro, A. (2019a): “Emoji and rapport management in Spanish WhatsApp chats", en Journal of Pragmatics, n 143, pp.109-120.

Sampietro, A. (2019b): "Cómo hacer palabras con emojis: sustitución y enfatización visual de vocablos en WhatsApp" en REDD, $\mathrm{n}^{\circ} 2$, pp. 1-32.

Steingress-Carballar, N. (2019): "Self y selfie en la época de su reproductibilidad técnica", en Universitas Humanística, $\mathrm{n}^{\circ}$ 87, pp. 121-141.

Sutton, B., \& Borland, E. (2017): "El discurso de los derechos humanos y la militancia por el derecho al aborto en la Argentina", en XIII Jornadas de Historia de las Mujeres y VIII Congreso Iberoamericano de Estudio de Género, pp. 24-27. Disponible en Internet: https://www.researchgate.net/publication/324760049_El_discurso_de_ los_derechos_humanos_y_la_militancia_por_el_derecho_al_aborto_en_la_Argentina [Consulta: 14 de diciembre de 2019].

Vela Delfa, C. (2007): El correo electrónico el nacimiento de un nuevo género. Tesis doctoral. Universidad Complutense de Madrid: Madrid.

Vela Delfa, C. (2014). Estrategias de construcción y mantenimiento de la imagen pública en canales de chat. Aposta. Revista de Ciencias Sociales, n 63, pp. 1-28.

Yus, F. (2014): "Not all emoticons are created equal”, en Linguagem em (Dis) curso, $n^{\circ} 14$, vol. 3, pp. 511-529. 
136 